\title{
Lower frequency of focal lip sialadenitis (focus score) in smoking patients. Can tobacco diminish the salivary gland involvement as judged by histological examination and anti-SSA/Ro and anti-SSB/La antibodies in Sjögren's syndrome?
}

R Manthorpe, C Benoni, L Jacobsson, Z Kirtava, Å Larsson, R Liedholm, C Nyhagen, $\mathrm{H}$ Tabery, E Theander

Sjögren's Syndrome Research Centre, Division of

Rheumatology, Department of Internal Medicine, Malmö University Hospital, S-205 02

Malmö, Sweden

R Manthorpe

L Jacobsson

C Nyhagen

E Theander

Sjögren's Syndrome Research Centre, Division of

Gastroenterology, Department of Internal Medicine C Benoni

National Information Learning Centre of the Ministry of Health of

Georgia and

Department of Clinical Pharmacology, Tbilisi

State Medical

University, Tbilisi,

Georgia

Z Kirtava

Sjögren's Syndrome Research Centre, Department of Oral Pathology, Centre for Oral Health Sciences Å Larsson

Sjögren's Syndrome Research Centre, Department of Oral Surgery and Oral Medicine, Centre for Oral Health Sciences R Liedholm

Sjögren's Syndrome Research Centre, Department of Ophthalmology H Tabery

Correspondence to: Dr R Manthorpe

Accepted for publication 26 August 1999

\begin{abstract}
Objectives-Prospectively collected computer database information was previously assessed on a cohort of 300 patients who fulfilled the Copenhagen classification criteria for primary Sjögren's syndrome. Analysis of the clinical data showed that patients who smoked had a decreased lower lip salivary gland focus score $(p<0.05)$. The aim of this original report is to describe the tobacco habits in patients with primary Sjögren's syndrome or stomatitis sicca only and to determine if there is a correlation between smoking habits and focus score in lower lip biopsies as well as ciculating autoantibodies and IgG.
\end{abstract}

Methods-All living patients with primary Sjögren's syndrome or stomatitis sicca only, who were still in contact with the Sjögren's Syndrome Research Centre were asked to fill in a detailed questionnaire concerning present and past smoking habits, which was compared with smoking habits in a sex and age matched control group $(n=3700)$ from the general population. In addition, the patients previous lower lip biopsies were blindly re-evaluated and divided by the presence of focus score (focus score $=$ number of lymphocyte foci per $4 \mathrm{~mm}^{2}$ glandular tissue) into those being normal (focus score $\leq 1$ ) or abnormal (focus score $>1$ ). Furthermore the cohort was divided into three groups; $10-45,46-60$ and $\geqslant 61$ years of age. Finally the focus score was related to the smoking habits. Seroimmunological (ANA; anti-SSA/Ro antibodies; antiSSB/La antibodies; IgM-RF and IgG) samples were analysed routinely.

Results-The questionnaire was answered by $98 \%(n=355)$ of the cohort and the percentage of current smokers, former smokers and historical non-smokers at the time of lower lip biopsy was not statistically different from that of the control group.
Cigarette smoking at the time of lower lip biopsy is associated with lower risk of abnormal focus score $(p<0.001$; odds ratio $0.29,95 \% \mathrm{CI} 0.16$ to 0.50 ). The odds ratio for having focal sialadenitis (focus score > 1) compared with having a non-focal sialadenitis or normal biopsy (focus score $\leqslant 1$ ) was decreased in all three age groups (10-45: odds ratio $0.27,95 \%$ CI 0.11 to 0.71 ; 46-60: odds ratio $0.22,95 \% \mathrm{CI} 0.08$ to 0.59 ; and $\geqslant 61$ : odds ratio $0.36,95 \%$ CI 0.10 to 1.43) although there was only statistical significance in the two younger age groups. Moreover, among current smokers at the time of the lower lip biopsy there was a decreasing odds ratio for an abnormal lip focus score with increasing number of cigarettes smoked per week ( $p$ trend 0.00 ). In the group of former smokers, which included patients that had stopped smoking up to 30 years ago, the results were in between those of the smokers and the historical non-smokers (odds ratio $0.57,95 \% \mathrm{CI} 0.34$ to 0.97 , compared with never smokers). Present or past smoking did not correlate with the function of the salivary glands as judged by unstimulated whole sialometry, stimulated whole sialometry or salivary gland scintigraphy. Among former smokers, the median time lapse between the first symptom of primary Sjögren's syndrome and the performance of the lower lip biopsy was approximately half as long as the median time lapse between smoking cessation and biopsy (8 versus 15 years). Hence, symptoms of Sjögren's syndrome are unlikely to have had a significant influence on smoking habits at the time of the biopsy. Among the seroimmunological results only anti-SSA/Ro and antiSSB/La antibodies reached statistical significance in a manner similar to the way smoking influenced the focus score in lower lip biopsies. On the other hand the level of significance was consistently more 
pronounced for the influence of smoking on the focus score than for the influence on anti-SSA/Ro and anti-SSB/La autoantibodies.

Conclusion-This is believed to be the first report showing that cigarette smoking is negatively associated with focal sialadenitis-focus score $>1$-in lower lip biopsy in patients with primary Sjögren's syndrome. Furthermore, tobacco seems to decrease the focus score in a dose dependent manner. Smoking may also negatively influence the presence of antiSSA/Ro and/or anti-SSB/La antibodies in circulating blood. Thus, smoking habits of patients might invalidate the use of both lower lip salivary gland focus score and of anti-SSA/anti-SSB antibodies. It is suggested that the simultaneous performance of other objective tests is required to avoid misdiagnosis of oral involvement in smoking and former smoking patients. Therefore, classification criteria for Sjögren's syndrome that more or less rely on an abnormal focus score and/or presence of anti-SSA/anti-SSB antibodies should be used with great caution.

(Ann Rheum Dis 2000;59:54-60)

Smoking is generally considered to be a great health hazard leading to increased socioeconomic expense and premature death. In contrast, some immunological disorders seem to be negatively associated with smoking. These include aphthous stomatitis, ${ }^{1}$ extrinsic alveolitis, sarcoidosis and hay fever (reviewed by Baron $^{2}$ ).

In ulcerative colitis, smoking seems to have a beneficial effect as smokers have a reduced risk and former smokers an increased risk of developing the disease. ${ }^{3}$ The reason for this protective effect is not known. Nicotine has proved effective only when added to standard treatments in moderately active disease ${ }^{4}$ and not in maintaining remission. ${ }^{5}$

A negative association such as a protective effect of smoking seems also to exist for younger cases of Parkinson's disease ${ }^{6}$ while advancing age showed a significant positive trend association. ${ }^{6}$ Both in Parkinson's and Alzheimer's diseases the nicotine system involvement may lead to new treatment strategies. $^{78}$

Primary Sjögren's syndrome (SS) is a systemic rheumatological disorder with a frequency that seems to exceed that of other systemic rheumatic diseases. The criteria for diagnosis may vary from country to country as no worldwide consensus exists, and no single diagnostic test has yet been found. Since 1976, we have used the Copenhagen criteria, ${ }^{9}$ which is one of seven existing classification methods, to diagnose primary SS.

When analysing the clinical observations of 300 patients with primary SS and a further 30 patients with only stomatitis sicca prospectively collected over the past 12.5 years, we found that smokers had a reduced frequency of abnormal lower lip focal sialadenitis or focus score (focus score $=$ number of lymphocyte foci per $4 \mathrm{~mm}^{2}$ glandular tissue; normal focus score $\leqslant 1$ ) compared with non-smokers (unpublished data). To evaluate if this effect was dependent on the dose of tobacco or on the initiation or termination of smoking, we mailed our present patients a questionnaire concerning past and present tobacco habits. In addition the patients' previous lower lip biopsy data were blindly re-evaluated.

The purpose of this report is to describe the tobacco habits in patients with primary SS or stomatitis sicca only and to determine if there is a correlation between smoking habits and focus score in lower lip biopsies as well as circulating autoantibodies and IgG. The null hypothesis stated that there was an equal percentage of focal sialadenitis (focus score $>1$ ) in the lower lip biopsy among smoking and non-smoking patients with primary SS.

\section{Methods}

PATIENTS AND DEFINITIONS

Since late 1984 we have prospectively computerised symptoms, signs, haematological, serological and immunological data from patients fulfilling the Copenhagen classification criteria for primary SS. ${ }^{9}$ Diagnosis was based on at least two abnormal objective tests for the lachrymal glands (keratoconjunctivitis sicca) and at least two abnormal objective test for the salivary glands (stomatitis sicca). In patients with normal lower lip focus score $(\leqslant 1)$ at least two other objective oral tests should be abnormal before the requirement for stomatitis sicca is fulfilled-most often unstimulated whole sialometry (abnormal if $\leq 1.5 \mathrm{ml} / 15 \mathrm{~min}$ ) and salivary gland scintigraphy, but a few times sialography was used.

As our prospective data did not contain a detailed tobacco history, we mailed a questionnaire (see below) to those patients still being seen at our SS Research Centre. New patients were asked to fill in the smoking questionnaire as well.

SMOKING QUESTIONNAIRE

The formula had the following questions:

(1) Are you a current smoker? If yes, (a) Do you smoke regularly? or (b) irregularly? Note: As many patients consider themselves as only feast smokers, both subgroups should state the year they started and the number of cigarettes (or packages) smoked per week.

(2) Are you a former smoker? If yes, (a) What year did you start? (b) What year did you stop? (c) How many cigarettes (or packages) did you smoke on average per week? (d) Did you use nicotine chewing gum and/or patches to reduce stopping? (e) Are you still using nicotine chewing gum and/or patches? If yes, In what amounts?

(3) Have you ever smoked?

(4) Do you take snuff? If yes, How many grams are you using per week?

For questions 1 and 2, the patients had the option to report pipe, cigars and/or cheroots instead of cigarettes. 
Table 1 Smoking habits of patients with primary Sjögren's syndrome or stomatitis sicca only at the time of lower lip biopsy, divided into three different age groups, and compared with control group

\begin{tabular}{|c|c|c|c|c|c|c|c|}
\hline \multirow[b]{2}{*}{ Year of age at the time of biopsy } & \multirow[b]{2}{*}{$10-45$} & \multirow[b]{2}{*}{$46-60$} & \multirow[b]{2}{*}{$\geqslant 61$} & \multirow[b]{2}{*}{ Total } & \multicolumn{3}{|c|}{ Smoking habits for: } \\
\hline & & & & & SS patients & & Controls \\
\hline Never smoked with valid biopsy & 42 & 45 & 68 & 155 & & & \\
\hline $\begin{array}{l}\text { Never smoked with non-valid biopsy } \\
\text { Never smoked and never biopsy } \\
\text { Current smokers with valid biopsy } \\
\text { Current smokers with non-valid biopsy }\end{array}$ & $\begin{array}{l}3 \\
5 \\
28 \\
0\end{array}$ & $\begin{array}{l}3 \\
5 \\
25 \\
1\end{array}$ & $\begin{array}{l}3 \\
6 \\
8 \\
1\end{array}$ & $\begin{array}{l}9 \\
16 \\
61 \\
2\end{array}$ & $\begin{array}{l}180= \\
51 \%\end{array}$ & $\begin{array}{l}\text { Historical } \\
\text { non-smokers }\end{array}$ & $50 \%$ \\
\hline $\begin{array}{l}\text { Current smokers and never biopsy } \\
\text { Former smokers who smoked at the time of lip biopsy }\end{array}$ & $\begin{array}{l}1 \\
8\end{array}$ & $\begin{array}{l}1 \\
6\end{array}$ & $\begin{array}{l}1 \\
4\end{array}$ & $\begin{array}{l}3 \\
18\end{array}$ & $\begin{array}{l}84= \\
24 \%\end{array}$ & Current smokers & $28 \%$ \\
\hline Former smokers who stopped before the time of lip biopsy & 20 & 37 & 34 & 91 & $\begin{array}{l}91= \\
25 \%\end{array}$ & Former smokers & $22 \%$ \\
\hline Total number of patients and controls & 107 & 123 & 125 & 355 & 355 & $\mathrm{p}=0.141$ & 3700 \\
\hline
\end{tabular}

SS patients = primary Sjögren's syndrome plus stomatitis sicca only patients. Never smoked = historical non-smokers (see text).

Patients were asked to return their answer in a prepaid stamped envelope. If no answer was received after two months, the patients received a telephone call or a second reminder letter.

CONTROLS

During 1993-94, 2000 randomly selected women and 2000 randomly selected men living in Malmö (250 000 inhabitants) and born 1913, 1923, 1933, 1943, 1953, 1963, 1968 and 1973 participated in a health study. ${ }^{10}$ Between $75 \%$ and $80 \%$ reported about their smoking habits and these were divided into three groups: historical non-smokers (= never smokers and feast smokers), former smokers and current smokers.

As $90 \%$ of patients with primary SS and stomatitis sicca only are women we composed a sex matched control group with a 9:1 female: male ratio for each 10 year period within the total 21-81 years of age to evaluate smoking habits in normal controls. The few patients with primary SS who were younger than 21 or older than 81 years of age were, for statistical purposes, considered to be 21 or 81 years of age, respectively.

FOCAL SIALADENITIS IN LOWER LIP BIOPSIES

To determine an accurate focus score, the previous lower lip biopsies were blindly reevaluated by our specialist in oral pathology (ÅL). A full description was performed and focal sialadenitis was expressed as lymphocytic foci per defined area of each salivary tissue specimen. Thereupon, the focus score of lymphocytic foci $/ 4 \mathrm{~mm}^{2}$ was calculated. A cut off focus score $>1$ was defined as abnormal although it occasionally might occur in normal or non-Sjögren's patients with other diseases.

IMMUNOLOGICAL DATA

Serum/plasma samples for the analyses of the following immunological data were taken at subsequent visits: anti-nuclear antibodies (ANA); anti-SSA (Ro 52 and 60) antibodies; anti-SSB (La 48) antibodies; IgM-RF; IgG. All analyses were performed routinely at the university hospital clinical chemistry or medical microbiology laboratories.

During the first years the ANA analyses were performed by immunofluorescence using rat liver sections as antigen. From 1992 the HEp-2 cells were used as antigen substrate. For women titres $\geqslant 64$; $\geqslant 128$ or $\geqslant 256$ were considered positive in the age groups $\leqslant 45$ years; $46-60$ years or $\geqslant 61$ years, respectively. For men one titre lower in the corresponding age group was considered positive.

IgM-RF was analysed by the classic haemagglutination method or by the agglutination method using plastic particles as substrate. Titres $\geqslant 64$ by the haemagglutination method or $\geqslant 80$ by the agglutination method were considered positive.

Anti-SSA (Ro 52 and/or 60) and anti-SSB (La 48) antibodies were analysed by immunodiffusion and results were registered either positive or negative.

For $\mathrm{p}-\mathrm{IgG}$ values $\geq 14.9 \mathrm{~g} / 1$ were considered equivalent with hyper-IgG-globulinaemia.

\section{STATISTICS}

Statistical calculations were done using the $\chi^{2}$ test, Mann-Whitney U non-parametric or Kruskal-Wallis test. p Values $<0.05$ were considered significant.

\section{Results}

MATERIAL SIZE

Our computerised patient material consists of information from 386 patients of whom 35 had stomatitis sicca only. Twenty four of the 386 patients had died or have no further contact with our Research Centre. A total of 362 smoking questionnaires were thus distributed to the patients. At the time of diagnosis $90.5 \%$ were women with median age 53.7 years (interquartile range: 42.7-61.7), while the median age for men was 48.3 years (interquartile range: 40.3-58.9).

ANSWERED SMOKING QUESTIONNAIRE

Eighty nine per cent of the patients returned the completed questionnaire without reminders. A further $9 \%$ returned it after a reminder or answered a telephone call. The cohort thus comprised 355 patients (98\%) with a valid answered smoking questionnaire. When presenting results, the cohort was subdivided into three age groups at the time of diagnosis: $10-45,46-60$ and $\geqslant 61$ years (tables 1 and 2). 
Table $2 \chi^{2}$ Tests for patients with primary Sjögren's syndrome or stomatitis sicca only divided into the three age groups $10-45,46-60$ and $\geqslant 61$ years of age as well as into \pm focal sialadenitis in lower lip biopsy, and into \pm smoking at the time of the biopsy. Only historical non-smokers and current smokers are included in the table

\begin{tabular}{|c|c|c|c|c|c|c|c|c|}
\hline & \multicolumn{2}{|c|}{ All patients Sialadenitis } & \multicolumn{2}{|c|}{$\begin{array}{l}\text { 10-45 years old } \\
\text { Sialadenitis }\end{array}$} & \multicolumn{2}{|c|}{$\begin{array}{l}46-60 \text { years old } \\
\text { Sialadenitis }\end{array}$} & \multicolumn{2}{|c|}{$\begin{array}{l}\geqslant 61 \text { years old } \\
\text { Sialadenitis }\end{array}$} \\
\hline & Focal & $\begin{array}{l}\text { Non-focal- } \\
\text { or normal }\end{array}$ & Focal & $\begin{array}{l}\text { Non-focal- } \\
\text { or normal }\end{array}$ & Focal & $\begin{array}{l}\text { Non-focal- } \\
\text { or normal }\end{array}$ & Focal & $\begin{array}{l}\text { Non-focal - } \\
\text { or normal }\end{array}$ \\
\hline \multirow[t]{2}{*}{ Never smoked } & 98 & 57 & 26 & 16 & 32 & 13 & 40 & 28 \\
\hline & $63 \%$ & $37 \%$ & $62 \%$ & $38 \%$ & $71 \%$ & $29 \%$ & $59 \%$ & $41 \%$ \\
\hline \multirow[t]{2}{*}{ Current smokers } & 26 & 53 & 11 & 25 & 11 & 20 & 4 & 8 \\
\hline & $33 \%$ & $67 \%$ & $31 \%$ & $69 \%$ & $35 \%$ & $65 \%$ & $33 \%$ & $67 \%$ \\
\hline Total & \multirow{2}{*}{\multicolumn{2}{|c|}{$\begin{array}{l}234 \\
\mathrm{p}<0.001\end{array}$}} & \multirow{2}{*}{\multicolumn{2}{|c|}{$\begin{array}{l}78 \\
\mathrm{p}=0.006\end{array}$}} & \multirow{2}{*}{\multicolumn{2}{|c|}{$\begin{array}{l}76 \\
\mathrm{p}=0.002\end{array}$}} & \multirow{2}{*}{\multicolumn{2}{|c|}{$\begin{array}{l}80 \\
\mathrm{p}=0.102\end{array}$}} \\
\hline $\mathrm{p}$ values & & & & & & & & \\
\hline Odds ratio $(95 \% \mathrm{CI})$ & \multicolumn{2}{|c|}{$0.29(0.16$ to 0.50$)$} & \multicolumn{2}{|c|}{$0.27(0.11$ to 0.71$)$} & \multicolumn{2}{|c|}{$0.22(0.08$ to 0.59$)$} & \multicolumn{2}{|c|}{$0.36(0.10$ to 1.43$)$} \\
\hline
\end{tabular}

Never smoked $=$ historical non-smokers $($ see text).

SMOKING HABITS

Historical non-smokers comprised 180 patients $(50.7 \%)$ and the proportion increased with age to $61.6 \%$ non-smokers among patients 61 years of age or older (table 1 ).

Former smokers comprised 109 patients $(30.7 \%)$ (table 1$)$. We checked to see if former smokers had been smoking at the time of the lower lip biopsy. If this was the case, the patient was re-categorised as a current smoker. Eighteen former smoking patients were thus recategorised as current smokers (table 1). There remained 91 former smoking patients (25.6\%), who, at any given time, had stopped smoking before the lower lip procedure.

Current smokers at the time of the biopsy thus comprised 66 plus 18 former smoking (see above) patients at the time of lower lip biopsy, or 84 patients $(23.7 \%)$ (table 1 ).

Of the 175 former plus current smoking patients who once had started smoking, most had begun at the age of 18 years (interquartile range: $17-21$ ).

Statistical calculations showed no difference in smoking habits among patients with primary SS at the time of diagnosis and controls (table 1) and stopping smoking in the group of patients with primary SS showed no bimodal distribution.

CIGARETTES VERSUS OTHER TYPES OF SMOKING None of the smoking patients smoked tobacco products other than cigarettes. Although snuff is a popular tobacco habit among Swedish persons, in this investigation only eight male patients reported using snuff.

Nicotine chewing gum and/or patches were used only by some of the former smokers once they stopped smoking and none of these were continuous users.

Table 3 Average number of cigarettes smoked per week in 84 patients with primary Sjögren's syndrome or stomatitis sicca only who are current smokers at the time of lower lip salivary gland biopsy, compared with the 180 patients who never smoked. The material is further divided into focal (focus score >1) or non-focal sialadenitis in the biopsy

\begin{tabular}{llllll}
\hline Cigarettes per week & $\begin{array}{l}\text { Focal } \\
\text { sialadenitis }\end{array}$ & $\begin{array}{l}\text { Non-focal } \\
\text { sialadenitis or normal }\end{array}$ & $\begin{array}{l}\text { None or } \\
\text { non-valid biopsy }\end{array}$ & Odds ratio & $95 \%$ CI \\
\hline $0=$ never smoked & 98 & 57 & 25 & 3.5 & 2.0 to 6.2 \\
$1-7$ & 3 & 1 & 0 & 1.7 & 0.2 to 16.8 \\
$8-21$ & 4 & 2 & 0 & 1.2 & 0.2 to 6.6 \\
$22-50$ & 7 & 11 & 0 & 0.4 & 0.1 to 1.0 \\
$51-100$ & 6 & 24 & 3 & 0.2 & 0.1 to 0.4 \\
$101-200$ & 6 & 13 & 2 & 0.3 & 0.1 to 0.7 \\
$201-280$ & 0 & 2 & 0 & 0.1 & 0.0 to 1.5 \\
Total & 26 & 53 & 5 & & \\
& & p for linear trend $=0.00$ & & \\
\hline
\end{tabular}

FOCUS SCORE IN LOWER LIP BIOPSIES AND THE INFLUENCE OF SMOKING

Table 2 shows that current smokers have a substantially reduced frequency of abnormal focus score in the lower lip biopsy when compared with patients who had never smoked $(\mathrm{p}<0.001 ;$ OR 0.29). In other words, the frequency of non-focal sialadenitis or normal biopsy findings, for example, focus score $\leq 1$ is increased in smokers. When dividing the data into the three age groups, the odds ratio for a positive biopsy was decreased in all age groups (10-45 years: $\mathrm{OR}=0.27 ; 46-60$ years: $\mathrm{OR}=$ $0.22 ; \geqslant 61$ years: $\mathrm{OR}=0.36)$, although this was only statistically significant in the two younger age groups (table 2).

CURRENT SMOKERS' CIGARETTE USE AT THE TIME OF LOWER LIP BIOPSY IN RELATION TO FOCAL OR NON-FOCAL SIALADENITIS

Table 3 shows the weekly cigarette consumption among the 79 current smokers with a valid biopsy. The maximum number was 280 cigarettes weekly.

The weekly average cigarette consumption was 64 in those with focal sialadenitis and 88 in those with non-focal sialadenitis. In the group of smokers, the odds ratio for having focal sialadenitis compared with non-focal sialadenitis decreased from 3.5 to 0.1 with increased number of cigarettes smoked per week ( $\mathrm{p}$ for linear trend $=0.00)$ (table 3 ).

For smokers there seems to be a threshold between focal (focus score $>1$ ) and non-focal sialadenitis at around 21 cigarettes weekly - the odds ratio being $<1$ if the cigarette consumption is more than 21 weekly (table 3 ).

LOWER LIP BIOPSY OF FORMER SMOKERS IN RELATION TO FOCAL OR NON-FOCAL

SIALADENITIS

When the 91 former smokers had the results of the lower lip biopsy divided into focal sialadenitis (focus score $>1$ ) and non-focal sialadenitis the frequency of an abnormal biopsy was in between the frequency observed in current and historical non-smokers (table 2) with an odds ratio $0.57,95 \% \mathrm{CI} 0.34$ to 0.97 , compared with never smokers (data not shown).

TIME LAPSE BETWEEN STOPPING SMOKING AND LOWER LIP BIOPSY AND THE INFLUENCE ON FOCAL SIALADENITIS

Based upon the 91 former smokers (table 1) we calculated the median time lapse between 
stopping smoking and lower lip biopsy to be 15 years (interquartile 7-22).

The time lapse between stopping smoking and lower lip biopsy result appeared similar in the two groups (median for focal sialadenitis: 14 years; median for non-focal sialadenitis including normal biopsy: 15.5 years; $\mathrm{p}=0.46$ ).

TIME LAPSE BETWEEN FIRST SJÖGREN'S SYMPTOM AND LOWER LIP BIOPSY (TIME AT DIAGNOSIS)

The median and interquartiles time lapse (in years) was not statistically different among current, $(7(3-11))$ former $(8(4-14))$ or historical non-smokers (5 (3-9)).

There were also no significant differences with regard to such time lapse when comparing the groups with focal $(10(4-17))$ and nonfocal sialadenitis $(8(4-15))$.

UNSTIMULATED WHOLE SIALOMETRY, STIMULATED WHOLE SIALOMETRY, SALIVARY GLAND SCINTIGRAPHY, SIALOGRAPHY AND THE INFLUENCE OF SMOKING

No significant differences between current smokers, former smokers or historical nonsmokers with regard to the above three oral functional tests or sialography (only performed in 16 patients) could be observed (data not shown).

FOCAL SIALADENITIS (FOCUS SCORE $>1$ ) VERSUS NON-FOCAL OR NORMAL LOWER LIP BIOPSY IN RELATION TO THE PRESENCE OF ANA, ANTI-SSA/RO ANTIBODIES, ANTI-SSB/LA ANTIBODIES, IgM-RF AND IgG VALUES IN SERUM/PLASMA

Positivity or negativity for the variables ANA, IgM-RF and p-IgG showed no statistical significant correlation $(p>0.05)$ in relation to focal versus non-focal or normal sialadenitis.

Sixteen per cent of the smokers and $32 \%$ of the historical non-smokers had anti-SSA antibodies ( $p<0.01$; odds ratio 0.40 (95\% CI: 0.20 to 0.81$)$ ). Former smokers had values in between. In all the three age groups the upper $95 \%$ odds ratio confidence limit was $>1.0$.

Patients with anti-SSA antibodies had more often focal sialadenitis $(85 \%)$ than non-focal or normal sialadenitis $(15 \%)(\mathrm{p}=0.001)$. The $\chi^{2}$ test between \pm focal sialadenitis and historical non-smokers and current smokers showed significance $(\mathrm{p}<0.02)$ with odds ratio 0.14 (95\% CI: 0.03 to 0.75 ).

Patients without anti-SSA antibodies had more often non-focal or normal sialadenitis than focal sialadenitis $(\mathrm{p}<0.02)$. The $\chi^{2}$ test between \pm focal sialadenitis and historical nonsmokers and current smokers showed significance $(\mathrm{p}<0.01)$ with odds ratio $0.38(95 \% \mathrm{CI}$ : 0.19 to 0.74 ).

Eleven per cent $(11 \%)$ of the smokers were seropositive and $25 \%$ of the historical nonsmokers had anti-SSB antibodies $(p<0.02$; odds ratio 0.35 (95\% CI: 0.16 to 0.81$)$ ). Former smokers had values in between. The upper $95 \%$ odds ratio confidence limit was > 1.0 for the youngest and oldest patient groups while the $46-60$ years old had odds ratio 0.11 (95\%CI: 0.01 to 0.95 ).

\section{Discussion}

In patients with primary SS and in patients with stomatitis sicca only, cigarette smokers seem to have a significantly lower frequency of abnormal glandular focus score in lower lip biopsy. Our findings also clearly show a dose response relation between an abnormal focus score and the number of cigarettes smoked (table 3), whereas the effect seems to be unrelated to age. Our main conclusion is therefore, that in smokers, the cigarette products have a negative influence on the development of foci in patients who otherwise fulfill the diagnosis of primary SS.

To what extent can there be bias in this investigation? Our patient material has been systematically collected and registered since the end of 1984 . We are the only referral centre for the 250000 inhabitants of Malmö as well as for its closest surroundings. However, approximately $25 \%$ of the patients diagnosed with primary SS or with stomatitis sicca only are referred from other parts of Sweden. The investigational procedures performed by the same Sjögren's experts have, however, been identical during this period of time. Any major selection bias is therefore unlikely.

The Copenhagen classification criteria for the diagnosis of primary SS including stomatitis sicca have been used since $1984 . .^{9}$ These criteria, which were developed during 1975/76, imply that for the diagnosis of primary SS, at least two abnormal objective test results for the function of the lachrymal glands (keratoconjunctivitis sicca), as well as at least two abnormal objective test results for the function of the salivary glands (stomatitis sicca), are required. ${ }^{9}$ Thus all our patients with a pathological focus score have at least one, and over $80 \%$ have two, other pathological salivary gland test results. The patients with non-focal sialadenitis (focus score $\leqslant 1$ ) - or with no valid or performed lower lip biopsy-always had two other abnormal salivary gland test results.

Most other classification criteria used for the diagnosis of primary SS require only one abnormal test result from the lachrymal and/or salivary glands to establish keratoconjunctivitis sicca and/or stomatitis sicca. Therefore, other Sjögren centres may have difficulty confirming our observations unless such objective tests are performed at the time of diagnosis. At many centres, most clinicians will perform lower lip biopsy as one of the first investigational procedures and will not always perform additional clinical investigations if non-focal sialadenitis or normal salivary gland biopsy is found (focus score $\leqslant 1 / 4 \mathrm{~mm}^{2}$ salivary gland tissue). We suggest that, if clinicians do not take into consideration the patients' tobacco habits (cigarettes), a focus score $\leqslant 1$ may be a false negative in a smoking patient. If smoking habits differ among potential patients or countries, results of clinical and laboratory data among various Sjögren centres could be expected to differ, particularly if there are also differences in classification criteria and evaluation procedures.

According to the newest classification criteria for primary SS, the Japanese II criteria, ${ }^{11}$ a 
patient should be classified as having primary SS as soon as an abnormal salivary gland focus score can be demonstrated in a lower lip biopsy. Many departments in USA use the California (or San Diego) criteria of Fox et $a l^{12}$ to diagnose the oral component of SS in which abnormal focus score is required as a conditio sine qua non. At our centre a young or middle aged patient classified according to the Copenhagen criteria as having primary SS or stomatitis sicca only and who smokes more than 21 cigarettes per week will have about $70 \%$ chances of a "negative or normal" lower lip biopsy. As long as clinicians lack single test results that are both specific and sensitive, diagnostic or classification criteria should rely upon the combination of two or more abnormal objective test results. When judging the oral component of SS more objective test results than lower lip biopsy alone ought to be available.

As inclusion criteria to the examined cohort is based on having abnormal findings in at least two objective oral tests any association between smoking habits and salivary gland functional tests other than lower lip biopsy could introduce a bias. The lack of association between smoking habits and unstimulated whole sialometry, stimulated whole sialometry and salivary gland scintigraphy does not support such bias. Furthermore, the oral pathologist was not aware of the smoking habits of the patients before the histopathological examination of the small salivary glands. This also supports the notion that it is not possible to judge the function of the big salivary glands based upon the histopathological picture in the small salivary glands taken from the lower lip.

At the time of the lower lip biopsy, which in our cohort corresponds to the time of diagnosis, there were no statistically significant differences between patients with primary SS and the control group concerning the frequency of current smokers, former smokers and historical non-smokers. This might be surprising as it is generally accepted that symptoms of dry mouth and also dry eyes are exaggerated by smoke. Although current smokers affected by primary SS at our Research Centre do admit that smoke might increase their oral/ocular symptoms this worsening is quite acceptable in patients addicted to cigarettes. Furthermore, as some smoking patients view the situation: "It is not the smoke from my own cigarettes which worsens my oral/ocular complaint but the smoke from the persons close to me"!

Could the more seriously ill patients have stopped smoking because of discomfort and thus reveal more serious salivary gland involvement? Although, our smoking questionnaire did not tackle this question, in former smoking patients the median difference between stopping smoking and the diagnostic (including lower lip biopsy) procedure was 15 years, while the median difference between the first Sjögren symptom and the primary SS diagnosis was eight years. Hence, initial symptoms of SS are unlikely to have had significant influence on quitting smoking at the time of the lip biopsy.
Again, this supports our clinical experience that stopping smoking is a sudden impulse occurring at a special date that the former smoker always remembers (unpublished data).

As the negative association between smoking and an abnormal focus score in lower lip biopsy could also be observed in the former smoking group, which included subjects who had stopped smoking up to three decades ago, we hypothesise that in some patients the protective effect of smoking lasts for several years after smoking cessation.

It would be convincing if our patient material had included a group of patients who were historical non-smokers at the time of diagnosing primary SS with abnormal lower lip focus score, and then had a second normal (focus score $\leqslant 1$ ) lower lip biopsy taken years after starting smoking. We did not have such patients and doubt that other research centres have such a representative group of patients.

The biochemical explanation for the reduced abnormal lower lip focus score in smokers is unknown at present. However, our results are similar to those observed in patients suffering from ulcerative colitis, aphthous stomatitis and sarcoidosis, other diseases where smoking has been shown to have some positive influence. In both of the oral gastrointestinal disorders, nicotine is the pharmacological compound most likely to be responsible (see introduction).

Among the various immunological parameteres in peripheral blood smoking stastistically influenced only the presence of antiSSA/Ro antibodies and anti-SSB/La antibodies in a manner similar to the effect on focal sialadenitis in lower lip biopsy. The results, on the other hand, are less pronounced than the negative association between smoking and focus score in lower lip biopsy.

Previous investigations have shown that the anti-SSA/Ro antibodies and anti-SSB/La antibodies are mainly produced by lymphocytes/ plasma cells in the diseased salivary glands, ${ }^{13}$ and there is a strong positive correlation between serum levels of these autoantibodies and the number of antibody producing cells within the salivary glands. ${ }^{13}$ Another study on patients with primary SS have shown a positive correlation between focus score in lower lip biopsy and presence of anti-SSA/Ro and anti-SSB/La antibodies in circulating blood. ${ }^{14}$ This is in good agreement with our observation that $85 \%$ of patients who are positive for antiSSA/Ro antibodies have focal sialadenitis. In other words the focus score in lower lip biopsy and the presence of anti-SSA/Ro antibodies and/or anti-SSB/La antibodies in peripheral blood are not independent variables.

The convincing smoking results from this large monocentre investigation combined with the knowledge of which cells accumulate and what they produce in the exocrine salivary glands support the view that smoking lowers the focus score by reducing the accumulation of lymphocytes/plasma cells in exocrine salivary glands. This will lead secondarily to lesser production of autoantibodies by the inflammatory cells in the salivary glands, which thirdly is 
measured as normal levels of anti-SSA/Ro antibodies and/or anti-SSB/La antibodies in peripheral blood.

In conclusion, cigarette smoking is associated with a reduced glandular focus score in lower lip biopsy among patients suffering from primary SS or stomatitis sicca, diagnosed according to the Copenhagen criteria. There is a clear cigarette dose dependency (table 3) as the focus score decreased with the number of cigarettes smoked per week, and reflected by a decreasing odds ratio. Cigarette smoking likewise - but to a lesser degree - reduced antiSSA/Ro antibodies and anti-SSB/La antibodies in peripheral blood. This supports our hypothesis that smoking lowers the focus score by reducing the accumulation of lympocytes/ plasma cells in lower lip biopsy (salivary glands). This secondarily leads to lesser production of anti-SSA/Ro antibodies and anti-SSB/La antibodies by the inflammatory cells in the salivary glands, which thirdly is measured as normal levels of anti-SSA/Ro antibodies and/or anti-SSB/La antibodies in peripheral blood.

Our observations further support the view that the focus score in lower lip biopsy and the presence of anti-SSA/Ro antibodies and/or anti-SSB/La antibodies in peripheral blood are not independent variables. This may be of great importance when diagnosing an individual patient. Classification criteria for primary SS that include functional test results from the exocrine glands should be used.

We acknowledge the valuable help of the statisticians Jan Åke Nilsson and Jonas Ranstam and Dr Marston Manthorpe for editorial comments. Supported by EU Biomed 2 contract BMH4-CT96-0595.
1 Axéll T, Henricsson V. Association between recurrent Axell T, Henricsson V. Association between recurrent aphthous ulcers

2 Baron JA. Beneficial effects of nicotine and cigarette smoking: the real, the possible and the spurious. $\mathrm{Br}$ Med Bull 1996;52:58-73.

3 Benoni C, Nilsson A. Smoking habits in patients with inflammatory bowel disease. A case-control study. Scand J Gastroenterol 1987;22:1130-6.

4 Pullan RD, Rhodes J, Ganesh S, Mani V, Morris JS, Williams GT, et al. Transdermal nicotine for active ulcerative colitis. N Engl J Med 1994;330:811-15.

5 Thomas GAO, Rhodes J, Mani V, Williams GT, Newcombe RG, Russell MAH, et al. Transdermal nicotine as maintenance therapy for ulcerative colitis. N Engl J Med 1995;332:988-92

6 Tzourio C, Rocca WA, Breteler MM, Baldereschi M, Dartigues JF, Lopez-Pousa S, et al. Smoking and Parkinson's disease. An age dependent risk effect? The 1267-72.

7 Newhouse PA, Potter A, Levin ED. Nicotinic system involvement in Alzheimer's and Parkinson's diseases. involvement in Alzheimer's and Parkinson's diseases. 28 .

8 Ott A, Slooter AJC, Hofman A, van Harskamp F, Witteman JCM, van Broeckhoven C, et al. Smoking and risk of dementia and Alzheimer's disease in a population based cohort study: the Rotterdam Study. Lancet 1998;351: 1840-3.

9 Manthorpe R, Oxholm P, Prause JU, Schiødt M. The Copenhagen criteria for Sjögren's syndrome. Scand J Rheumatol 1986; suppl 61:19-21.

10 Lindström $M$, Bexéll A, Hansson BS, Isacsson S-O. Hälsoläget i Malmö: Rapport från post enquete undersökning, 1994:33. [In Swedish]

11 Fujibayashi T, Sugai S, Miyasaka N, Tojo T, Miyawaki S, Ichikawa Y, et al. Preliminary revision of the Japanese criteria for Sjögren's syndrome. J Rheumatol 1997;24 (suppl 50):38.

12 Fox RI, Robinson C, Curd J, Michelson P, Bone R, Howell FV. First International Symposium on Sjögren's Syndrome: Suggested Criteria for Classification. Scand J Rheumatol 1986;suppl 61:28-30. [Later also published in: Arthritis Rheum 1986;29:577-85.]

13 Tengnér P, Halse A-K, Haga H-J, Jonsson R, WahrenHerlenius $M$. Detection of anti-Ro/SSA and anti-La/SSB autoantibody-producing cells in salivary glands from patients with Siögren's syndrome. Arthritis Rheum 1998; patients with $41: 2238-48$.

14 Gerli R, Muscat C, Giansanti M, Danieli MG, Sciuto M, Gabrielli A, et al. Quantitative assessment of salivary gland inflammatory infiltration in primary Sjögrens's syndrome: its relationship to different features of the disorder. Br J Rheumatol 1997;36:969-75. 